\title{
Análise interobservador da classificação das lesões em dedo em martelo segundo Albertoni, utilizando-se radiografias simples: Importância do uso do goniômetro*
}

\section{Interobserver Analysis of Albertoni Classification for Mallet Finger, Using Plain Radiography: Importance of the Goniometer}

\author{
Tomás Santos Vasconcelos Barros ${ }^{1}$ [0 Antônio José Jeha ${ }^{1} \quad$ Guilherme Vieira Borchio Ribeiro ${ }^{1}$ \\ ${ }^{1}$ Departamento de Ortopedia, Hospital Vera Cruz, Belo Horizonte, \\ MG, Brasil \\ Endereço para correspondência Tomás Santos Vasconcelos Barros, \\ MD, Hospital Vera Cruz, Av. Barbacena, 653, Barro Preto, Belo \\ Horizonte, MG 30190-130, Brasil \\ Rev Bras Ortop 2020;55(2):198-202. \\ (e-mail: tomasbarros@hotmail.com).
}

\section{Resumo}

Palavras-Chave

- deformidades adquiridas da mão/ classificação

- traumatismos dos dedos

- reprodutibilidade dos testes

- ruptura

- traumatismos dos tendões
Objetivos Quantificar o grau de concordância intra- e interobservador da classificação Albertoni e avaliar a importância do uso do goniômetro na diferenciação do grau da lesão.

Métodos Foram selecionados 10 casos de dedo em martelo, os quais foram avaliados por 60 examinadores.

Resultados A concordância interobservador sem o uso do goniômetro foi elevada. Com o uso do goniômetro, obteve-se um "kappa" ainda maior, porém sem relevância estatística.

Conclusão A Classificação de Albertoni possui elevada concordância intra- e interobservador, e o uso do goniômetro se mostrou dispensável para classificar.

Objectives The objective of the present study is to evaluate the intraobserver and interobserver reliability of the Albertoni classification for mallet finger. Evaluation of goniometer device application is also an objective.

Methods A total of 10 lateral radiographs of patients with mallet finger were selected and measured by 60 orthopedic surgeons with and without the use of goniometer. Results The intra- and interobserver reliability coefficients found were high. With the use of a goniometer, the interobserver reliability coefficient was even higher, but without statistical relevance.

Conclusion The Albertoni classification showed high intraobserver and interobserver reliability in assessing mallet finger lesions, and the goniometer is dispensable for this purpose.

Trabalho feito no Hospital Vera Cruz, Belo Horizonte, MG, Brasil.

recebido

27 de Dezembro de 2019

Aceito

08 de Janeiro de 2019
DOI https://doi.org/

$10.1055 / \mathrm{s}-0039-3400518$. ISSN $0102-3616$
Copyright $\odot 2020$ by Sociedade Brasileira License terms de Ortopedia e Traumatologia. Published by Thieme Revinter Publicações Ltda, Rio de Janeiro, Brazil 


\section{Introdução}

O dedo em martelo tem por definição a ruptura do mecanismo extensor terminal, resultando em uma deformidade em flexão da falange distal. ${ }^{1}$

As lesões do mecanismo extensor são muito frequentes nos traumatismos da mão. 0 terceiro, quarto e quinto dedos da mão dominante são os mais acometidos, sendo que $95 \%$ das lesões são fechadas. $O$ mecanismo de lesão em geral é um trauma na ponta do dedo provocando flexão súbita ou extensão contra-resistência. ${ }^{1,2}$

Esta lesão, quando não tratada, poderá resultar em um tendão alongado e potencialmente menos funcional. A ruptura da cápsula e dos ligamentos retinaculares leva a uma deformidade maior. É importante ressaltar que as fibras do ligamento retinacular oblíquo também possuem papel na extensão. As lesões ósseas por avulsão provocam deformidades semelhantes pela desinserção junto ao fragmento das estruturas tendinosas e ligamentares. ${ }^{2-4}$

A presente pesquisa tem como objetivo geral quantificar a concordância intra- e interobservador da classificação Albertoni, e avaliar a importância do uso do goniômetro nas lesões do mecanismo extensor terminal do dedo ${ }^{3,5,6}$ ( - Figura 1 ).

\section{Materiais e Métodos}

O protocolo do estudo foi aprovado pelo comitê de ética institucional sob o número CAAE 57854216300005135.

\section{Classificação de Albertoni para dedo em martelo}

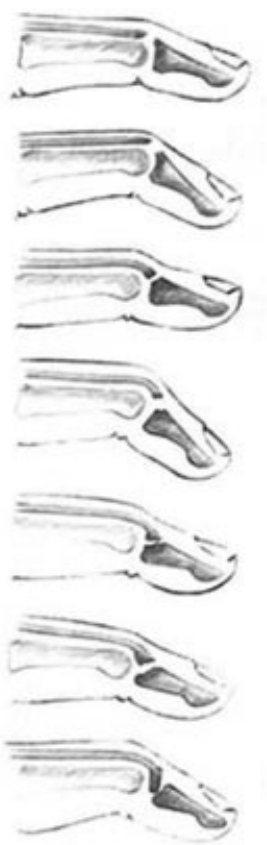

A1. Lesão tendínea pura com queda da falange distal $<30^{\circ}$.

A2. Lesâo tendínea pura com queda da falange distal em $30^{\circ}$.

B1. Lesão com arrancamento ósseo e queda da falange distal $<30^{\circ}$.

B2. Lesão com arrancamento ósseo e queda da falange distal em $30^{\circ}$.

C1. Lesão com fratura da base da falange distal com a articulação interfalangeana distal estável.

C2. Lesão com fratura da base da falange distal com a articulação interfalangeana instável.

D1. Deslocamento epifisário da falange distal.

Fig. 1 Esquema demonstrando a classificação proposta por Albertoni (Omitimos os tipos C e D para enfatizarmos a aferição do ângulo). Fonte: Albertoni et al. ${ }^{7}$
Trata- se de um estudo observacional transversal realizado entre os dias 08 e 22 de agosto de 2016. Foram selecionados 10 casos com lesões de dedo em martelo para serem examinadas através de radiografias de incidência em perfil, com e sem o uso do goniômetro. Todas as imagens foram digitalizadas e então analisadas por ortopedistas e residentes em ortopedia que atuam nos serviços de saúde públicos e privados da cidade de Belo Horizonte, MG, Brasil, e a região metropolitana. $O$ questionário é dividido em duas partes, cada uma contendo dez questões e um cabeçalho com informações sobre a formação do profissional (Material Suplementar 1 ).

Omitimos os tipos C e D da classificação de Albertoni para enfatizarmos a aferição do ângulo e o uso do goniômetro. Nos tipos $C$ e D, a angulação não é essencial para a classificação (além disso, são lesões mais raras).

Em um dos questionários, as radiografias deveriam ser analisadas sem o uso de goniômetro (SG) e no outro com o uso desse instrumento (CG). Os critérios de inclusão de profissionais são: possuir familiaridade com o uso do goniômetro e o conhecimento da classificação de Albertoni para dedo em martelo. As radiografias foram avaliadas por 60 profissionais, sendo 20 residentes de ortopedia, 32 ortopedistas, e 8 cirurgiões de mão.

Para descrever o ângulo entre as questões e entre as classificações das questões, foram utilizadas medidas de tendência central, dispersão e posição. Para verificar a concordância intra e interobservador foi utilizado o coeficiente de Kappa. ${ }^{7,8}$

O software utilizado na análise foi o $\mathrm{R}$ versão 3.2.2.

\section{Resultados}

Foram aplicados 60 questionários. Destes, dois questionários foram excluídos por não estarem completos ou por possuírem os ângulos medidos com letras ilegíveis.

A - Tabela 1 apresenta os observadores quanto à formação “Cirurgia da Mão". Oito (13\%) dos respondentes eram especialistas em cirurgia de mão.

A - Tabela 2 apresenta a descrição das classificações das questões com e sem uso do goniômetro. O percentual de concordância de resposta foi elevado, sendo que nas questões 2 e 8 a maioria foi classificada como "A", nas questões 3,4 e 5 a maioria foi classificada como "B", nas questões 1, 6, 7 e 9 a maioria foi classificada como " $\mathrm{C}$ ", e na questão 10 a maioria foi classificada como "D".

A - Figura 2 a seguir ilustra as informações da tabela acima.

A - Tabela 3 apresenta a média do ângulo medido pelos entrevistados.

Tabela 1 Descrição das variáveis subespecialidade e formação

\begin{tabular}{|l|l|l|l|}
\hline \multicolumn{2}{|c|}{} & $n$ & $\%$ \\
\hline $\begin{array}{l}\text { Especialidade em } \\
\text { Cirurgião da mão }\end{array}$ & Não & 50 & $86 \%$ \\
\cline { 2 - 4 } & Sim & 8 & $13 \%$ \\
\hline
\end{tabular}


200 Análise interobservador da classificação das lesões em dedo em martelo segundo Albertoni Barros et al.

Tabela 2 Descrição das Classificações das Questões Sem e Com uso do Goniômetro

\begin{tabular}{|c|c|c|c|c|c|c|c|c|c|c|c|c|c|c|c|c|}
\hline \multirow[t]{3}{*}{ Questões } & \multicolumn{8}{|c|}{ Questões sem uso do Goniômetro } & \multicolumn{8}{|c|}{ Questões com uso do Goniômetro } \\
\hline & \multicolumn{2}{|l|}{ A1 } & \multicolumn{2}{|l|}{$\mathrm{A} 2$} & \multicolumn{2}{|l|}{ B1 } & \multicolumn{2}{|l|}{ B2 } & \multicolumn{2}{|l|}{ A1 } & \multicolumn{2}{|l|}{$A 2$} & \multicolumn{2}{|l|}{ B1 } & \multicolumn{2}{|l|}{ B2 } \\
\hline & $n$ & $\%$ & $n$ & $\%$ & $n$ & $\%$ & $n$ & $\%$ & $n$ & $\%$ & $n$ & $\%$ & $n$ & $\%$ & $n$ & $\%$ \\
\hline Questão 1 & 0 & $0,0 \%$ & 0 & $0,0 \%$ & 58 & $100,0 \%$ & 0 & $0,0 \%$ & 0 & $0,0 \%$ & 0 & $0,0 \%$ & 57 & $100,0 \%$ & 0 & $0,0 \%$ \\
\hline Questão 2 & 58 & $100,0 \%$ & 0 & $0,0 \%$ & 0 & $0,0 \%$ & 0 & $0,0 \%$ & 54 & $94,7 \%$ & 3 & $5,3 \%$ & 0 & $0,0 \%$ & 0 & $0,0 \%$ \\
\hline Questão 3 & 0 & $0,0 \%$ & 57 & $98,3 \%$ & 1 & $1,7 \%$ & 0 & $0,0 \%$ & 0 & $0,0 \%$ & 56 & $98,2 \%$ & 0 & $0,0 \%$ & 1 & $1,8 \%$ \\
\hline Questão 4 & 7 & $12,1 \%$ & 46 & $79,3 \%$ & 1 & $1,7 \%$ & 4 & $6,9 \%$ & 4 & $7,0 \%$ & 52 & $91,2 \%$ & 0 & $0,0 \%$ & 1 & $1,8 \%$ \\
\hline Questão 5 & 1 & $1,7 \%$ & 56 & $96,6 \%$ & 1 & $1,7 \%$ & 0 & $0,0 \%$ & 1 & $1,8 \%$ & 56 & $98,2 \%$ & 0 & $0,0 \%$ & 0 & $0,0 \%$ \\
\hline Questão 6 & 1 & $1,7 \%$ & 0 & $0,0 \%$ & 54 & $93,1 \%$ & 3 & $5,2 \%$ & 2 & $3,5 \%$ & 0 & $0,0 \%$ & 53 & $93,0 \%$ & 2 & $3,5 \%$ \\
\hline Questão 7 & 0 & $0,0 \%$ & 1 & $1,7 \%$ & 55 & $94,8 \%$ & 2 & $3,4 \%$ & 1 & $1,8 \%$ & 0 & $0,0 \%$ & 55 & $96,5 \%$ & 1 & $1,8 \%$ \\
\hline Questão 8 & 55 & $94,8 \%$ & 2 & $3,4 \%$ & 1 & $1,7 \%$ & 0 & $0,0 \%$ & 55 & $96,5 \%$ & 2 & $3,5 \%$ & 0 & $0,0 \%$ & 0 & $0,0 \%$ \\
\hline Questão 9 & 0 & $0,0 \%$ & 4 & $6,9 \%$ & 54 & $93,1 \%$ & 0 & $0,0 \%$ & 0 & $0,0 \%$ & 0 & $0,0 \%$ & 56 & $98,2 \%$ & 1 & $1,8 \%$ \\
\hline Questão 10 & 0 & $0,0 \%$ & 0 & $0,0 \%$ & 0 & $0,0 \%$ & 58 & $100,0 \%$ & 0 & $0,0 \%$ & 0 & $0,0 \%$ & 0 & $0,0 \%$ & 57 & $100,0 \%$ \\
\hline
\end{tabular}

Questões sem uso

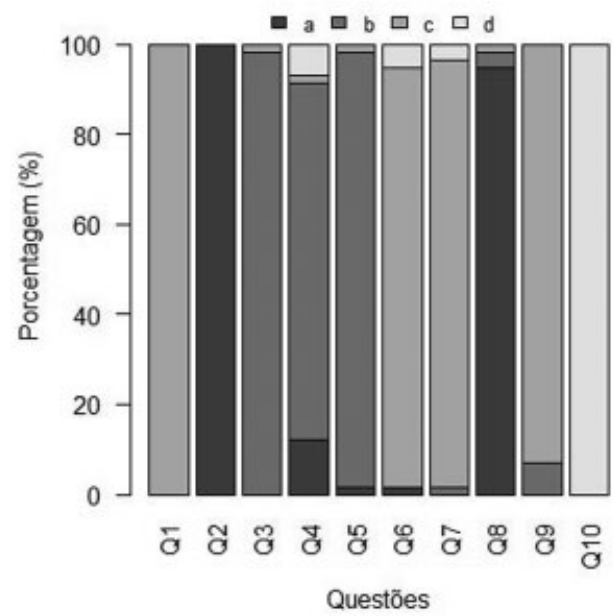

Questões com uso

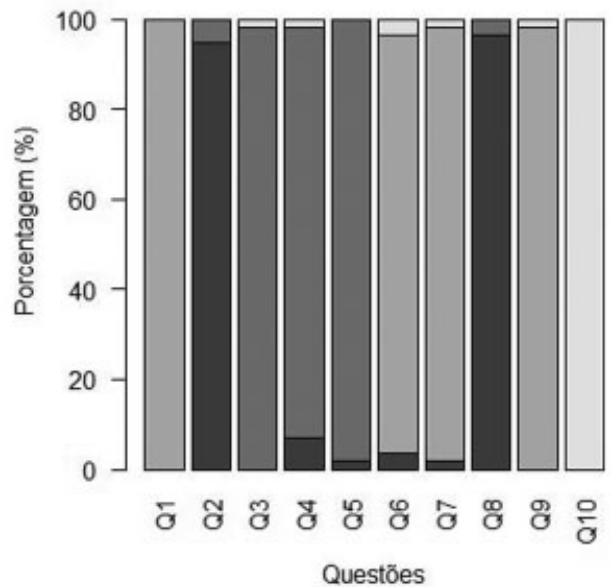

Fig. 2 Descrição das classificações das questões sem e com uso.

Tabela 3 Descrição do ângulo entre as questões

\begin{tabular}{|l|l|l|l|l|l|l|l|}
\hline Ângulo & Média & DP & Mín. & $1^{\circ}$ Quartil & $2^{\circ}$ Quartil & $3^{\circ}$ Quartil & Máx. \\
\hline Questão 1 & 18,32 & 3,78 & 10,00 & 16,00 & 20,00 & 20,00 & 28,00 \\
\hline Questão 2 & 22,47 & 4,15 & 13,00 & 20,00 & 21,00 & 26,00 & 35,00 \\
\hline Questão 3 & 40,95 & 4,01 & 30,00 & 40,00 & 40,00 & 44,00 & 50,00 \\
\hline Questão 4 & 35,35 & 4,90 & 26,00 & 32,00 & 35,00 & 40,00 & 48,00 \\
\hline Questão 5 & 35,47 & 4,49 & 28,00 & 32,00 & 35,00 & 39,00 & 49,00 \\
\hline Questão 6 & 21,53 & 3,49 & 10,00 & 20,00 & 20,00 & 22,00 & 32,00 \\
\hline Questão 7 & 9,86 & 4,64 & 2,00 & 8,00 & 10,00 & 11,00 & 35,00 \\
\hline Questão 8 & 17,46 & 5,46 & 10,00 & 13,00 & 18,00 & 20,00 & 32,00 \\
\hline Questão 9 & 11,79 & 4,58 & 4,00 & 10,00 & 10,00 & 45,00 & 48,00 \\
\hline Questão 10 & 44,33 & 4,62 & 34,00 & 40,00 & & 58,00 \\
\hline
\end{tabular}

Abreviações: DP, desvio padrão. 


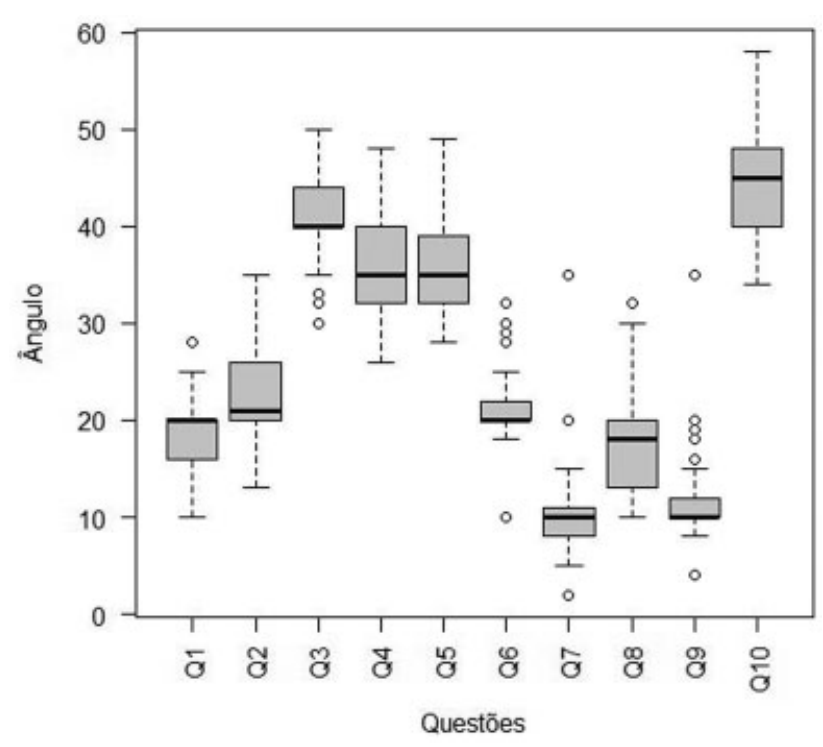

Fig. 3 Descrição do ângulo entre as questões.

A - Figura 3 a seguir ilustra em gráfico as informações da tabela acima.

A - Tabela 4 apresenta a concordância intraobservador e interobservador para cada questão. A partir dela, pode-se destacar que a concordância foi alta para todas as questões, sendo que:

- Com relação à concordância intraobservador ("com goniômetro" versus "sem goniômetro"), as questões 1 e 10 apresentaram uma concordância de $100 \%$, enquanto que a questão 4 apresentou a menor concordância. 0 coeficiente de Kappa apresentou um valor de 0,906, que indica uma alta concordância $(p=0,000)$.

Tabela 4 Concordância intraobservador e interobservador para cada questão

\begin{tabular}{|c|c|c|c|c|c|c|}
\hline \multirow[t]{3}{*}{ Questão } & \multirow{2}{*}{\multicolumn{2}{|c|}{$\begin{array}{l}\text { Intraobser- } \\
\text { vador }\end{array}$}} & \multicolumn{4}{|c|}{ Interobservador } \\
\hline & & & \multicolumn{2}{|c|}{ Sem uso } & \multicolumn{2}{|c|}{ Com uso } \\
\hline & $n$ & $\%$ & $n$ & $\%$ & $n$ & $\%$ \\
\hline Questão 1 & 57 & $100,0 \%$ & 58 & $100,0 \%$ & 57 & $100,0 \%$ \\
\hline Questão 2 & 54 & $94,7 \%$ & 58 & $100,0 \%$ & 54 & $94,6 \%$ \\
\hline Questão 3 & 55 & $96,5 \%$ & 57 & $98,2 \%$ & 56 & $98,2 \%$ \\
\hline Questão 4 & 45 & $78,9 \%$ & 46 & $79,0 \%$ & 52 & $91,1 \%$ \\
\hline Questão 5 & 54 & $94,7 \%$ & 56 & $96,5 \%$ & 56 & $98,2 \%$ \\
\hline Questão 6 & 53 & $93,0 \%$ & 54 & $93,0 \%$ & 53 & $92,9 \%$ \\
\hline Questão 7 & 53 & $93,0 \%$ & 55 & $94,7 \%$ & 55 & $96,4 \%$ \\
\hline Questão 8 & 52 & $91,2 \%$ & 55 & $94,7 \%$ & 55 & $96,4 \%$ \\
\hline Questão 9 & 52 & $91,2 \%$ & 54 & $93,0 \%$ & 56 & $98,2 \%$ \\
\hline Questão 10 & 57 & $100,0 \%$ & 58 & $100,0 \%$ & 57 & $100,0 \%$ \\
\hline Kappa & \multicolumn{2}{|c|}{0,906} & \multicolumn{2}{|c|}{0,829} & \multicolumn{2}{|c|}{0,865} \\
\hline Valor-p & \multicolumn{2}{|c|}{0,000} & \multicolumn{2}{|c|}{0,000} & \multicolumn{2}{|c|}{0,000} \\
\hline
\end{tabular}

\section{Discussão}

A importância e eficiência de uma classificação se baseiam na simplicidade e reprodutibilidade. A mesma deve ainda permitir a comparação entre estudos científicos e apresentar alta concordância entre examinadores. ${ }^{9,10}$ Ao avaliar a confiabilidade e concordância entre observadores, há necessidade de considerar o acaso na avaliação. ${ }^{11,12}$ Para tanto, é utilizado o método kappa de comparação. Foi incorporado também no presente trabalho o uso do goniômetro e sua influência sobre a concordância nessa classificação. ${ }^{11,13,14}$

A - Tabela 2 mostra a distribuição de respostas relativas a cada questão sem e com o uso do goniômetro. Vemos que as questões 1, 2, 3, 5, 6, 7, 8, 9 e 10 apresentaram baixa variação intra- e interobservador. A questão 4 apresentou maior discordância para uma mesma resposta. Isso é devido ao fato de essa questão demonstrar uma lesão em dedo martelo com medida limítrofe de classificação. ${ }^{15-17}$

Observou-se uma uniformidade entre as respostas obtidas, demonstrada por um desvio padrão com pouca variação entre as mesmas (entre $3,49^{\circ}$ e $5,46^{\circ}$ ). Na questão 8 , a falange distal apresentava uma deformidade, que pode ter afetado esses resultados isolados obtidos. No gráfico boxplot, que a variação de ângulos entre os quartis de cada questão foi pequena com uma media de $5,1^{\circ}$ (menor diferença: $2^{\circ} /$ maior diferença: $\left.8^{\circ}\right)$. Não foi encontrado trabalho como referência para comparação com esses dados. No entanto, consideramos os valores obtidos como adequados. ${ }^{18,19}$

Os resultados da avaliação intra- e interavaliador foram excelentes, como mostra a - Tabela 4, seguindo a Escala do Coeficiente Interclasse de Kappa (- Tabela 5).

Não houve diferença estatística relevante entre os avaliadores considerando a formação (residente, ortopedista ou cirurgião da mão). Lembrando que não foram incluídos os residentes que não conheciam a classificação ou que relataram não saber usar o goniômetro.

A concordância interavaliador para cada questão sem 0 uso do goniômetro com índice de kappa 0.829 (excelente) sugere que, mesmo sem o instrumento, a classificação é reprodutível para os diversos observadores. O uso do goniômetro gerou um kappa de 0.865 , ligeiramente superior, o que mostra que o goniômetro tem a capacidade de aumentar a concordância, porém sem relevância estatística. ${ }^{20-22}$

Tabela 5 Escala do Coeficiente Interclasse de Kappa

\begin{tabular}{|l|l|}
\hline Kappa & Interpretação \\
\hline$<0$ & Sem associação \\
\hline $0-0.19$ & Ruim \\
\hline $0.20-0.39$ & Baixa \\
\hline $0.40-0.59$ & Moderada \\
\hline $0.60-0.79$ & Boa \\
\hline $0.80-1$ & Excelente \\
\hline
\end{tabular}

Landis JR, Koch GG. The measurement of observer agreement for categorical data. Biometrics 1977; 33: 159-179. 
A medida de concordância intraobservador comparando os resultados obtidos com uso do goniômetro e sem o uso do mesmo apresentou um índice Kappa de 0,906. Por este trabalho, ficou estabelecido que a classificação não demanda o uso do goniômetro e que a avaliação por profissional que tenha conhecimento da classificação (residente de ortopedia, ortopedista ou cirurgião de mão) é suficiente.

\section{Conclusão}

Concluímos que a classificação de Albertoni é simples e reprodutível e com boa concordância intra- e interobservador, tanto com o uso do goniômetro, quanto sem ele. O uso do goniômetro aumentou um pouco a concordância interobservador, porém sem relevância estatística, sugerindo que é um instrumento dispensável para avaliação do dedo em martelo.

Conflito de Interesses

Os autores declaram não haver conflito de interesses.

\section{Referências}

1 Mélega JM. Cirurgia plástica fundamentos e arte: cirurgia reparadora de troncos e membros. Rio de Janeiro: Guanabara-Koogan; 2004

2 Braga Silva J. Cirurgia da mão - Trauma. Rio de Janeiro: Revinter; 2003

3 Young RE, Harmon JM. Repair of tendon injuries of the hand. Ann Surg 1960;151:562-566

4 Duran RJ, Houser RG. Controlled passive motion following flexor tendon repair in zone 2 and 3. In: AAOS Symposium on tendon surgery in the hand. St. Louis: CV Mosby; 1975. p. 105-14.

5 Lister GD, Kleinert HE, Kutz JE, Atasoy E. Primary flexor tendon repair followed by immediate controlled mobilization. J Hand Surg Am 1977;2(06):441-451

6 May EJ, Silfverskiöld KL, Sollerman CJ. Controlled mobilization after flexor tendon repair in zone II: a prospective comparison of three methods. J Hand Surg Am 1992;17(05):942-952
7 Albertoni WM, Leite VW. Lesões dos tendões extensores. In: Pardini Júnior AG. Traumatismo da mão. 3ª ed. Rio de Janeiro: Medsi; 2000:351-380

8 Fleiss J. The measurement of interrater agreement, statistical interrater agreement statistical. New York: John Wiley \& Sons; 1981

9 Braga Silva J, Martins PDE, Román J, Gehlen D. Mobilização pós-operatória com flexão ativa precoce após reparo de tendões flexores na zona. Rev Soc Bras Cir Plást. 2005;20(04): 207-212

10 Braga Silva J, Calcagnotto G, Oliveira CG, Fisher H. Estudo prospectivo randomizado da mobilização ativa precoce após reparo dos tendões flexores em zona 2. Rev Bras Ortop 2003;38(10): 581-588

11 Evans GR. Cirurgia plástica - estética e reconstrutora. Rio de Janeiro: Revinter; 2003

12 Braga Silva J, Fernandes H, Fridman M. Lesão do tendão extensor em zona 1: tratamento ortopédico e cirúrgico. Rev Bras Ortop 1999;34(02):139-144

13 Braga Silva J, Gazzalez A, Alvarez G. Lesão tendinosa da mão. Rev da AMRIGS, Porto Alegre. 2011;55(02):197-201

14 Barros Filho TE, Lech O. Exame físico em ortopedia. São Paulo: Sarvier; 2002

15 Browner B, Jupiter J, Levine A, Trafton P, Krettek C. Skeletal trauma. 4th ed. Philadelphia: Saunders; 2008

16 Canale ST, Beaty JH. Campbell's operative orthopaedics. 12th ed. St. Louis: Mosby; 2012

17 Hebert S, Barros Filho TE, Xavier R, Pardini Júnior AG. Ortopedia e traumatologia: princípios e prática. 5a . ed. Porto Alegre: Artmed; 2016

18 Morrissy RT, Weinstein SL. Lovell and Winter's pediatric orthopaedics. 4th ed. Philadelphia: Lippincott; 2005

19 Pardini Júnior AG, Freitas AD. Traumatismos da mão. 4ª ed. Rio de Janeiro: Medbook; 2008

20 Agresti A, Kateri M. Categorical data analysis. Berlin: SpringerVerlag; 2011

21 Braga-Silva J, Kuyven CR. Early active mobilization after flexor tendon repairs in zone two. Chir Main 2005;24(3-4):165-168

22 Hollander M, Wolfe DA, Chicken E. Nonparametric statistical methods. New York: John Wiley \& Sons; 2013 